\title{
Población y supervivencia fetal de cerdas pertenecientes a pequeñas explotaciones, por medio de material de rastro.
}

\author{
Comunicación Breve
}

Luis G. Montoya-Gómez, Ernesto Zorrilla-de la Torre, Francisco J. Escobar-Medina, Federico de la Colina-Flores.

Facultad de Medicina Veterinaria y Zootecnia de la Universidad Autónoma de Zacatecas. Zacatecas, México.

\section{RESUMEN.}

Introducción. Se estudió la capacidad de los ovarios para ovular, del útero para el desarrollo y la supervivencia fetal de las cerdas que se explotan alrededor de la Ciudad de Zacatecas y que pertenecen a pequeños productores.

Material y Métodos. El estudio se realizó con material de rastro en 50 cerdas criollas que se sacrificaron gestantes, con diferente número de partos, peso, edad y avance de la gestación. Al examen se anotó la longitud de los cuernos uterinos, el número de cuerpos lúteos en cada ovario y la cantidad, peso, tamaño y lugar de implantación de los productos.

Resultados. La media de la longitud del cuerno uterino derecho fue de $194.3 \pm 66.4 \mathrm{~cm}$ y $183.2 \pm$ $49.6 \mathrm{~cm}$ la del izquierdo. El promedio de cuerpos lúteos fue de $14.3 \pm 4.1$ en los dos ovarios. Se encontraron $7.9 \pm 2.6$ productos en los cuernos uterinos, $4.0 \pm 1.5$ en el derecho y $3.9 \pm 1.6$ en el izquierdo. No hubo efecto significativo en el tamaño de los productos con relación al lugar de su implantación ( $\mathrm{p}>0.05)$, pero la población fetal fue mayor en los dos tercios uterinos cercanos al oviducto, menor en el cercano al cuerpo del útero $(\mathrm{p}<0.05)$. Se encontraron $1.54 \pm 1.00$ fetos en tercio uterino más cercano al oviducto; $1.23 \pm 0.81$, en el intermedio, y $0.66 \pm 0.72$, en el extremo. La supervivencia fetal fue de $54.5 \%$, y de $62.0 \%$, $57.9 \%$ y $50.0 \%$ en las cerdas que se sacrificaron en el primer, segundo y tercer tercio de la gestación, respectivamente $(\mathrm{p}>0.05)$.

Discusión. Los resultados del presente trabajo sugieren que las cerdas criollas de este estudio tienen buena capacidad de ovulatoria, pero las gestaciones las desarrollan con baja supervivencia fetal. (Rev Biomed 2002; 13:185-188)

Palabras clave: Porcinos, cerdas, ovulación, supervivencia embrionaria.

Solicitud de sobretiros: Francisco J. Escobar-Medina, Facultad de Medicina Veterinaria y Zootecnia, Universidad Autónoma de Zacatecas. Carretera Panamericana, tramo Zacatecas - Fresnillo Km 31.5. Apdo. Postal 11, C.P. 98,500, Calera de Víctor Rosales, Zacatecas, México.

Fax (4) $9850202 . \quad$ E-mail fescobar@cantera.reduaz.mx 
LG Montoya-Gómez, E Zorrilla-de la Torre, FJ Escobar-Medina, F de la Colina-Flores.

\section{SUMMARY.}

Fetal numbers and survival in sows in the state of Zacatecas, Mexico.

Introduction. Ovulation ability and fetal survival and development was studied in sows on small farms located near the City of Zacatecas, Mexico. Materials and Methods. The study was carried out on organs obtained from 50 pregnant Creole sows slaughtered at the local abattoir, varying in weight and stage of gestation. The length of the uterine horns, the number of corpora lutea in each ovary, and the number, weight, size and location of the products in the uterus were recorded.

Results. The mean length of the uterine right horn was of $194.3 \pm 66.4 \mathrm{~cm}$ and $183.2 \pm 49.6 \mathrm{~cm}$ for the left one. The mean number of corpora lutea was of $14.3 \pm 4.1$ in both ovaries. $7.9 \pm 2.6$ fetuses were found in the uterine horns, $4.0 \pm 1.5$ in the right horn and $3.9 \pm 1.6$ in the left one. Size and weight of the products were not related to their location in the uterus $(p>0.05)$, but the fetal population was higher in the two thirds closer to the oviduct, than it was in the third closer to the uterus body $(\mathrm{p}<0.05) .1 .54 \pm 1.00$ fetuses were found in the closest to the oviduct third, $1.23 \pm$ 0.81 , in the middle third, and $0.66 \pm 0.72$ in the furthest third. Fetal survival rates were $54.5 \%$, and $62.0 \%, 57.9 \%$ and $50.0 \%$ in the sows slaughtered on the first, second and third stage gestation, respectively.

Discussion. The results of the present study suggest that the Creole sows' ovulation ability is similar to that of other genetic groups, but embryonic and fetal survival rates are smaller.

(Rev Biomed 2002; 13:185-188)

Key words: Swine, sows, ovulation, embryo survival.

\section{INTRODUCCIÓN.}

Por lo general, la cerda compromete la supervivencia de sus productos, particularmente la embrionaria. Esto se debe a que tiene la capacidad de ovular gran número de ovocitos, con un porcentaje de fertilización superior al $90 \%$ y no dispone del suficiente espacio uterino para desarrollarlos durante la gestación $(1,2)$. El resultado, una parte de los embriones no se implantan y mueren, y otros productos se pierden durante la vida fetal. El factor limitante, por lo tanto, es la capacidad de los cuernos uterinos.

Estos estudios se han realizado en las granjas con camadas numerosas, pero en la cerda criolla de Zacatecas, la que pertenece a productores pequeños, aún no se ha estudiado. El objetivo del presente trabajo fue determinar la capacidad de los ovarios para ovular y del útero para el desarrollo y supervivencia fetal en cerdas de explotaciones pequeñas, por medio de material de rastro.

\section{MATERIAL Y MÉTODOS.}

El trabajo se realizó con material del rastro municipal de Zacatecas, donde se sacrifican cerdos que en la región se conocen como criollos y pertenecen a pequeños productores de municipios cercanos a la Ciudad de Zacatecas.

Durante el mes de diciembre, se realizaron visitas periódicas para examinar los aparatos genitales de 50 hembras que se sacrificaron en ese rastro. No fue posible conocer la edad de los animales ni el número de partos que habían tenido. Los exámenes se realizaron inmediatamente después del sacrificio y, en cada caso, se registró el número de cuerpos lúteos en cada ovario y se contaron los productos presentes en cada cuerno uterino, así como su tamaño y peso. Se midió la longitud de los cuernos uterinos y se dividió entre tres. Se contó el número de productos en cada tercio. Se midió la distancia a la que se implantó cada feto con respecto a las trompas uterinas. La supervivencia fetal se determinó por medio de la razón número de fetos con características normales:número de cuerpos lúteos. El avance de la gestación se determinó con base en el criterio utilizado por Beker (3).

Las comparaciones en población fetal entre 


\section{Población y supervivencia fetal de cerdas.}

los diferentes tercios y cuernos del útero y para la sobrevivencia de los productos en los tres tercios de la gestación, se realizaron por medio de la prueba no paramétrica de Kruskal-Wallis. El peso y longitud cráneo-caudales de los fetos fue analizada por medio de un análisis de varianza con un diseño completamente aleatorizado.

\section{RESULTADOS.}

El $12 \%$ de las cerdas que se estudiaron se encontraban en el primer tercio de la gestación, el $46 \%$ en el segundo y el $42 \%$ en el tercero. Los promedios de la longitud de los cuernos uterinos, cantidad de cuerpos lúteos, población y supervivencia fetal se presentan en el cuadro 1 . El $44.9 \%$ de los productos se localizaron en el tercio del cuerno del útero cercano al oviducto, el 35.9\% en el tercio intermedio y el $19.2 \%$ en el cercano al cuerpo del útero $(\mathrm{p}<0.05)$. No se detectaron diferencias significativas $(p>0.05)$ entre los promedios del peso fetal de los tercios del útero cercano, medio y lejano al oviducto $(257.9 \mathrm{~g}, 233.4$ g y 230.8 g respectivamente). El tamaño de los productos, en el mismo orden fue de $8.3 \mathrm{~cm}, 8.3$ $\mathrm{cm}$ y $8.2 \mathrm{~cm}(\mathrm{p}>0.05)$.
La supervivencia fetal tendió a reducirse con el avance de la gestación, el $62.0 \%$ de los fetos se encontraron viables en las hembras que se sacrificaron en el primer tercio de la gestación, el $57.9 \%$ en las del segundo y $50.0 \%$ en las del tercer tercio. La diferencia no fue estadísticamente significativa $(\mathrm{p}>0.05)$.

\section{DISCUSIÓN.}

Los resultados del presente trabajo sugieren que las cerdas criollas de los municipios cercanos a la Ciudad de Zacatecas tiene la capacidad de ovular de la misma manera como lo hacen las cerdas de otros grupos genéticos, pero las gestaciones las desarrollan con menor supervivencia fetal.

Esta conclusión se basa en que en las cerdas de este estudio tenían en promedio 14.3 cuerpos lúteos, lo que coincide con los resultados de otras publicaciones. Por ejemplo, Wu y col. (5), independientemente al número de partos del animal, encontraron de 13.9 a 14.8 cuerpos lúteos. Sin embargo, las hembras del presente trabajo mostraron menor porcentaje de supervivencia fetal $(55.4 \%)$ con respecto al $77.3 \%$ y $60.9 \%$ reportados

Cuadro 1

Medias ( \pm D.E.) de la longitud de los cuernos uterinos $(\mathrm{cm})$, número de cuerpos lúteos en los ovarios, población y supervivencia fetal de 50 cerdas criollas gestantes, de las cuales 7, 22 y 21 se sacrificaron en el primer, segundo y tercer tercio de la gestación.

\begin{tabular}{llrc}
\hline \multirow{2}{*}{ Variable } & Media \pm D. E. & Mínimo & Máximo \\
\hline Longitud de los cuernos uterinos & $384.5 \pm 103.3$ & 223 & 610 \\
Derecho & $194.3 \pm 66.4$ & 62 & 365 \\
Izquierdo & $183.2 \pm 49.6$ & 58 & 290 \\
& & & \\
Número de cuerpos lúteos & $14.3 \pm 4.1$ & 8 & 28 \\
Ovario derecho & $7.1 \pm 2.5$ & 1 & 13 \\
Ovario izquierdo & $7.2 \pm 3.0$ & 3 & 13 \\
Población fetal & $7.9 \pm 2.6$ & 2 & 7 \\
Cuerno uterino derecho & $4.0 \pm 1.5$ & 1 & 7 \\
Cuerno uterino izquierdo & $3.9 \pm 1.6$ & & \\
& & & \\
Supervivencia fetal $\quad \%)$ & 55.4 & & \\
&
\end{tabular}




\section{LG Montoya-Gómez, E Zorrilla-de la Torre, FJ Escobar-Medina, F de la Colina-Flores.}

por Conejo (6) y Wu y col. (5), lo cual probablemente se debió a la menor longitud de los cuernos uterinos. La media de la longitud de los cuernos uterinos en este trabajo fue de $192.4 \mathrm{~cm}$, longitud menor a la encontrada por otros autores; Wu y col. (5) reportaron $266 \mathrm{~cm}$ y Conejo (6) 373.1 $\mathrm{cm}$ de longitud en los cuernos uterinos.

La parte del cuerno uterino donde se implantan los embriones es un factor importante para su desarrollo. Los productos que se implantan en el tercio cercano al oviducto generalmente presentan mayor incremento de tamaño y peso, que los del tercio cercano al cuerpo del útero; estos últimos generalmente mueren en el transcurso de la gestación o son muy pequeños al nacimiento. Los que se unen a la parte media del cuerno muestran crecimiento intermedio (2). En el presente estudio, el $44.7 \%$ de los productos se localizaron en el tercio cercano al oviducto, el $35.6 \%$ en el intermedio y el $19.7 \%$ en el tercio cercano al cuerpo del útero. Esto significa que el porcentaje de implantaciones fue más elevado en el lugar donde los productos, según los datos de la literatura (2), presentan mayor desarrollo; como consecuencia al aumentar la población fetal hubo menor espacio uterino, por lo que no se manifestaron diferencias significativas en el tamaño $(\mathrm{p}>0.05)$.

En este estudio, en el cual no fue posible conocer el número de partos ni la edad del animal, se encontró el $62 \%$ de supervivencia fetal durante el primer tercio de la gestación, lo cual concuerda con los resultados de la literatura, en donde se ha encontrado el $60 \%$ y el $67 \%$ de supervivencia al final del primer mes de la gestación en las cerdas primerizas y adultas, respectivamente $(2,7)$. En el presente trabajo, la supervivencia fetal tendió a reducirse conforme transcurrió la gestación, el $57.9 \%$ y $50.0 \%$ de los fetos se encontraron viables en las cerdas que se sacrificaron en el segundo y tercer tercio de la gestación, respectivamente. Esto significa que con relación al primer tercio de la preñez, la mortalidad fetal se incrementó $4.1 \%$ en el segundo tercio y $12.0 \%$ en el tercer tercio de la gestación. En cerdas adultas se ha

\section{Revista Biomédica}

reportado el $7 \%$ de mortalidad fetal entre 25 y 50 días de preñez (2).

En conclusión las cerdas criollas pertenecientes a pequeñas explotaciones cercanas a la Ciudad de Zacatecas presentan buena capacidad ovulatoria, como lo hacen animales de otros grupos genéticos, pero con menor supervivencia fetal, y los productos se desarrollan preferentemente en los dos tercios uterinos cercanos al oviducto.

\section{REFERENCIAS.}

1.- Anderson LL. Pigs. En: Hafez ESE, editor. Reproduction in Farm Animals. 5a ed. Philadelphia: Lea and Febiger; 1987. p. 324-44.

2.- Dziuk PJ. Reproduction in Pigs. En Cupps PT, editor. Reproduction in Domestic Animals. . San Diego CA: Academic Press Inc; 1991. p. 471-89.

3.- Beker K. Current knowledge on the substance and energy composition of pig fetuses and piglets. Anim Res Develop 1978; 8:30-55.

4.- SAS. SAS/STAT user's guide version 6. Cary NC: SAS Institute Inc; 1986.

5.- Wu MC, Hentzel MD, Dziuk PJ. Effects of stage of gestation, litter size and uterine space on the incidence of mummifice fetuses in pig. J Anim Sci 1988; 66:3202-7.

6.- Conejo JJJ. Crecimiento uterino por gestación temporal en cerdas primerizas y su efecto sobre el desarrollo del embrión y el tamaño de la camada. México (D.F.): Facultad de Medicina Veterinaria y Zootecnia de la U.N.A.M.; 1993.

7.- De Sea WP, Pleusaran P, Morcom CB, Dukelon WR. Exogenous steroid effects on litter size and early embryonic survival in swine. Theriogenology 1981; 15:245-55. 